\title{
UNCOUNTABLY MANY MILDLY WILD NON-WILDER ARCS ${ }^{1}$
}

\section{SAMUEL J. LOMONACO}

In [5] Fox and Harrold defined a Wilder arc as a mildly wild L.P.U. (locally peripherally unknotted) arc and then gave a complete classification of such arcs. In this paper, to show that the L.P.U. condition is essential, uncountably many mutually nonequivalent mildly wild non-L.P.U. arcs are constructed.

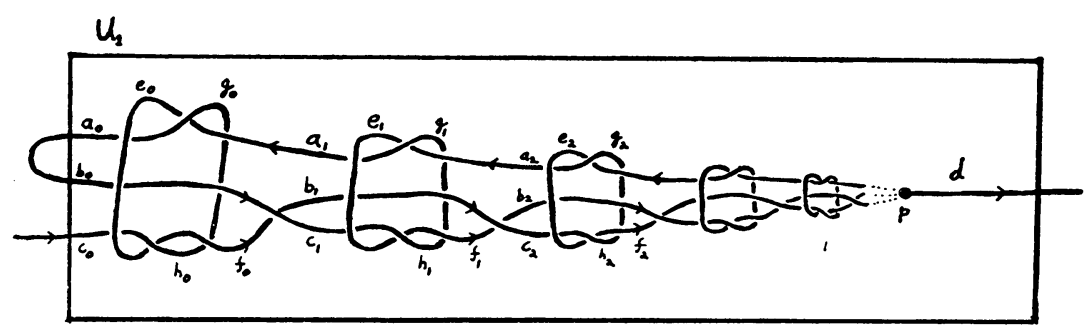

Figure 1. $A_{0}$

I. The basic example $A_{0}$ (Figure 1). A regular normed projection of our basic example of a mildly wild non-Wilder arc is shown in Figure 1. (Using the methods of [4], one could easily give a precise description.)

(A) $A_{0}$ is not L.P.U. at $p$. The invariants of [7] will be used to show that the penetration index $P\left(A_{0}, p\right)$ of $A_{0}$ at $p$ is equal to 4. (For a definition of the penetration index see [1] and [2].)

The fundamental group $\pi_{1}\left(U_{1}-A_{0}\right)$ of the complement of $A_{0}$ in the 3-cell neighborhood $U_{1}$ of $p$ is generated by elements $a_{n}, b_{n}, c_{n}, d$, $e_{n}, f_{n}, g_{n}, h_{n}(n \geqq 0)$ indicated in the usual way in Figure 1 . A set of defining relations is

$$
\begin{aligned}
g_{n} & =e_{n} a_{n} e_{n}^{-1}, \quad a_{n+1}=g_{n} e_{n} g_{n}^{-1}, \quad h_{n}=e_{n} c_{n} e_{n}^{-1}, \quad f_{n}=h_{n} e_{n} h_{n}^{-1}, \\
c_{n+1} & =e_{n} b_{n} e_{n}^{-1}, \quad a_{n+1} g_{n} a_{n+1}^{-1}=c_{n+1} f_{n} h_{n} \hat{f}_{n}^{-1} c_{n+1}^{-1}, \quad b_{n+1}=c_{n+1} f_{n} c_{n+1}^{-1}, \\
d & =a_{n}^{-1} b_{n} c_{n},
\end{aligned}
$$

where $n \geqq 0$. (The methods of [4] were used to find $\pi_{1}\left(U_{1}-A_{0}\right)$.)

Presented to the Society, August 29, 1967; received by the editors April 5, 1967.

1 This work was partially supported by the National Science Foundation Grant G-5458. 


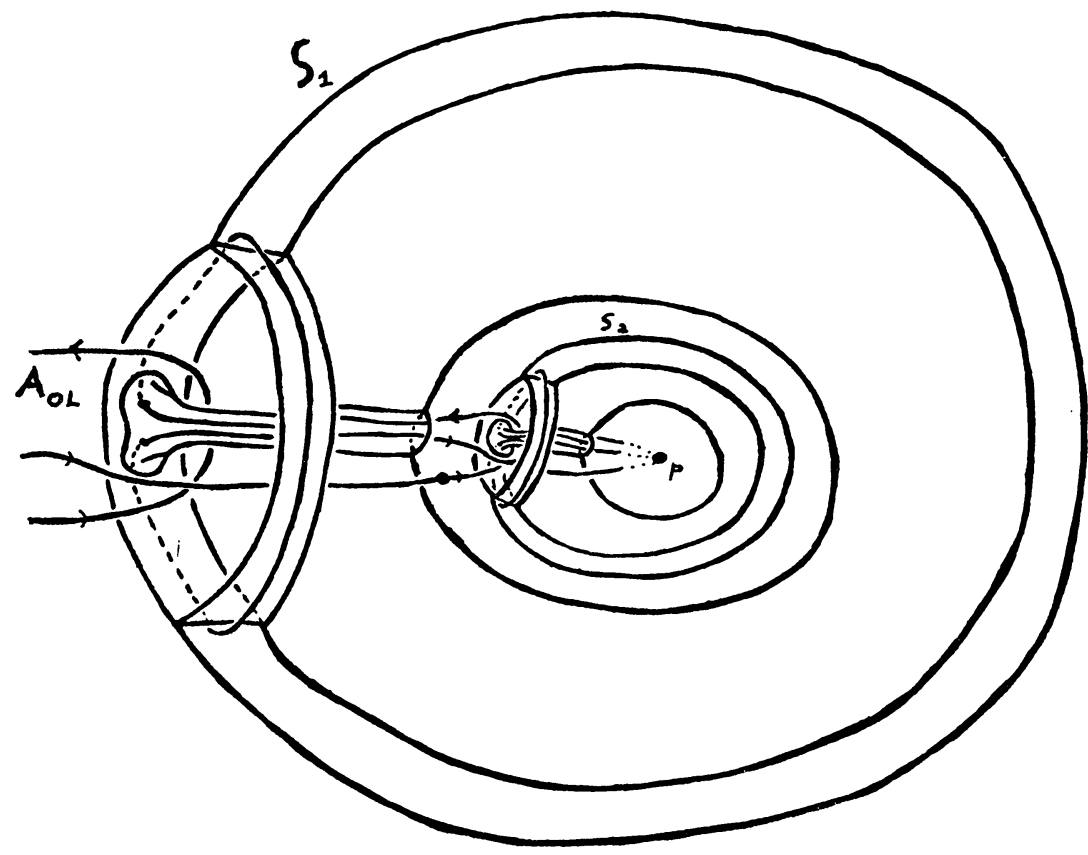

Figure 2

Simplifying the above presentation, one finds that $\pi_{1}\left(U_{1}-A_{0}\right)$ is generated by $a_{n}, c_{n}, e_{n}(n \geqq 0)$ with the following defining relations

$$
\begin{aligned}
e_{n} a_{n} e_{n} a_{n}^{-1} e_{n}^{-1} a_{n+1}^{-1} & =1, \\
e_{n}^{-1} a_{n+1}^{-1} c_{n+1} e_{n} c_{n} e_{n} c_{n} e_{n}^{-1} c_{n}^{-1} e_{n}^{-1} c_{n+1}^{-1} a_{n+1} e_{n} a_{n}^{-1} & =1, \\
e_{n+1} c_{n+1} e_{n} c_{n} e_{n} c_{n}^{-1} e_{n}^{-1} c_{n+1}^{-1} e_{n+1}^{-1} c_{n+2}^{-1} & =1,
\end{aligned}
$$

where $n \geqq 0$. Hence, the corresponding $J Z$-module is generated by $a_{n}, c_{n}, e_{n}(n \geqq 0)$ with the following corresponding defining relations:

$$
\left(t-t^{2}\right) a_{n}+\left(1-t+t^{2}\right) e_{n}-a_{n+1}=0 \quad(n \geqq 0),
$$

$-t^{2} a_{n}+\left(t^{2}-t^{3}+t^{4}\right) c_{n}+\left(t^{3}-t^{4}\right) e_{n}-(1-t) a_{n+1}+(1-t) c_{n+1}=0$, $\left(t^{3}-t^{4}\right) c_{n}+\left(t^{2}-t^{3}+t^{4}\right) e_{n}+\left(t-t^{2}\right) c_{n+1}+(1-t) e_{n+1}-c_{n+2}=0$,

where $t$ denotes the generator of the free cyclic group $Z$ and $J Z$ denotes the integral group ring of $Z$. Thus, the 0 th local topology $\Lambda_{0}\left(A_{0}, p\right)$ of $A_{0}$ at $p$ is $\Lambda_{0}\left(A_{0}, p\right)=(1-t)^{\infty}$.

From V.A.7 of [7] the appearance of the factor $1-t$ in $\Lambda_{0}\left(A_{0}, p\right)$ implies that $P\left(A_{0}, p\right)>2$, i.e. that $A_{0}$ is not L.P.U. at $p$. Moreover, since $P\left(A_{0}, p\right)$ must be even, it easily follows that $P\left(A_{0}, p\right)=4$. 
(B) $A_{0}$ is mildly wild.

That $A_{0}$ is actually mildly wild can be seen by constructing in the obvious fashion an isotopy taking the left half $A_{0 L}$ of $A_{0}$ onto a straight line segment. A more instructive but unfortunately more complicated method for showing that $A_{0 L}$ is tame is to observe that its penetration index [1], [2] at $p$ is equal to 1 . This can be seen from Figure 2 where a sequence of 2 -spheres converging to $p$ and intersecting $A_{0 L}$ at only one point is indicated.

II. Uncountably many examples. Let $\Delta_{1}(t), \Delta_{2}(t), \Delta_{3}(t), \cdots$ be an indexing of all mutually distinct (up to associates) prime polynomials in $t$ over the ring $J$ of integers such that:

(i) $\Delta_{i}(1)= \pm 1$

(ii) $\Delta_{i}(t)=t^{2 \lambda} \Delta(1 / t)$.

By [6], [8] there is a corresponding sequence of distinct knot types $K_{1}, K_{2}, K_{3}, \cdots$ whose Alexander polynomials are respectively $\Delta_{1}, \Delta_{2}, \Delta_{3}, \cdots$.

We may assume that each $K_{i}$ is a prime knot type. For if $K_{i}$ is not prime, then by [9], [10] it can be uniquely factored into a product of prime knot types, i.e.

$$
K_{i}=K_{i 1} \# K_{i 2} \# \cdots \# K_{i n},
$$

where each $K_{i j}$ is prime. Hence,

$$
\Delta_{i}=\Delta\left(K_{i}\right)=\Delta\left(K_{i 1}\right) \Delta\left(K_{i 2}\right) \cdots \Delta\left(K_{i n}\right),
$$

where $\Delta(K)$ denotes the Alexander polynomial of $K$. Since $J Z$ is a unique factorization domain and $\Delta_{i}(t)$ is prime, there must be a $j$ such that $\Delta\left(K_{i j}\right)=\Delta_{i}(t)$. Hence, $K_{i}$ may be replaced by the prime $K_{i j}$.

Let $S$ be the collection of all sequences of 0 or $\infty$, i.e. $S=\{0, \infty\}^{N}$, where $N$ denotes the set of natural numbers. For each $s$ in $S$ let $W_{s}$ be the Wilder arc in which each $K_{i}$ appears exactly $s(i)$ times $(i=1,2,3, \cdots)$ and no other knot types appear. (See [5].) Thus, by V.A.7 of [7] the 0th local topology $\Lambda_{0}\left(W_{\varepsilon}\right)$ of $W_{s}$ is

$$
\Lambda_{0}\left(W_{s}\right)=\prod_{i=1}^{\infty} \Delta_{i}^{s(i)}
$$

For each $s$ in $S$, let $A_{s}=A_{0} \$_{p} W_{s}$, where " $\$_{p}$ " denotes the interior composition of arcs defined in V.C.7 of [7]. By V.C.11 of [7],

$$
\Lambda_{0}\left(A_{s}\right)=\Lambda_{0}\left(A_{0}\right) \cdot \Lambda_{0}\left(W_{s}\right)=(1-t)^{\infty} \prod_{i=1}^{\infty} \Delta^{s(i)}
$$


(A) The $\operatorname{arcs} A_{s}$ are all mutually nonequivalent.

With the exponent defined in [7] it is easy to show that all the topologies $\Lambda_{0}\left(A_{s}\right)$ are distinct. Hence, by the Fundamental Invariance Theorem of [7] the arcs $A_{s}$ are all mutually nonequivalent.

(B) Not one of the arcs $A_{s}$ is L.P.U.

As in I.A. of this paper the appearance of $1-t$ in $\Lambda_{0}\left(A_{s}\right)$ implies that $P\left(A_{s}\right)=4$.

(C) Each of the arcs $A_{s}$ is mildly wild.

$A_{s}$ can be shown to be mildly wild in the obvious fashion. One can first apply an isotopy to the left half of $A_{s}$ to straighten out the $A_{0}$ factor and then another in turn to straighten out the $W_{s}$ factor. Since the right half of $A_{s}$ is the right half of a Wilder arc, it is also tame.

Hence, $\left\{A_{s}: s \in S\right\}$ is an uncountable collection of mutually nonequivalent mildly wild non-Wilder arcs.

III. Conjecture. The following conjecture is based on the examples in this paper as well as many others.

Conjecture. If $A$ is a mildly wild arc, then

$$
\Lambda_{0}(A)=(1-t)^{\infty} \prod_{i=1}^{\infty} \Delta_{i}(t)^{s(i)},
$$

where $\Delta_{i}(t)$ is a knot polynomial and $s(i)=0$ or $\infty$.

The author wishes to thank Charles Seebeck for simplifying the author's original example.

\section{REFERENCES}

1. W. R. Alford and B. J. Ball, Some almost polyhedral wild arcs, Duke Math. J., 30 (1963), 33-38.

2. B. J. Ball, "Penetration indices and applications" in Topology of 3-manifolds and related topics, Proc. Univ. of Georgia Inst., Prentice-Hall, Englewood Cliffs, N. J., 1962, pp. 37-39. 265.

3. R. H. Fox, A remarkable simple closed curve, Ann. of Math. (2) 50 (1949) 264-

4. R. H. Fox and E. Artin, Some wild cells and spheres in three-dimensional space, Ann. of Math. (2) 49 (1948), 979-990.

5. R. H. Fox and O. G. Harrold, "The Wilder arcs" in Topology of 3-manifolds and related topics, Proc. Univ. of Georgia Inst., Prentice-Hall, Englewood Cliffs, N. J., 1962, pp. 184-187.

6. J. Levine, A characterization of knot polynomials, Topology 4 (1965), 135-141.

7. S. Lomonaco, An algebraic theory of local knottedness. I, Trans. Amer. Math. Soc. 129 (1967), 322-343.

8. H. Seifert, Über das Geschlect von Knoten, Math. Ann. 110 (1934), 571-592.

9. H. Schubert, Die eindeutige Zerlegbarkeit eines Knotens in Primknoten, S.-B. Heidelberger Akad. Wiss. Math.-Nat. Kl. 3 (1949), 57-104.

10. - Knoten and Vollringe, Acta. Math. 90 (1953), 131-286.

Florida State University 